\title{
Special issue on Advances in MIMO-OFDM
}

The combination of Orthogonal Frequency Division Multiplexing (OFDM) and Multiple Input - Multiple Output (MIMO) techniques is instrumental to achieving the high spectral efficiency required for the evolution of mobile communication systems. The implementation of MIMO-OFDM systems will require, among others, advances in the design of channel estimation and synchronization techniques to work in fast fading conditions. These techniques in combination with strategies to manage or limit feedback information and overhead will allow for the adaptation of the transmitted signal to the changing channel conditions. MIMO-OFDM systems will use cooperative communication, through relays, to improve reliability and extend coverage.

The challenges faced when implementing MIMO-OFDM in the presence of multiple users increase, but also the benefits. Multiuser and multi-cell interference arise and resource allocation becomes crucial. Several approaches have been developed recently to improve the performance of MIMO-OFDM in multiuser and multi-cell environments, enhancing the data rates and/or the expanding the coverage.

The special issue on Advances in MIMO-OFDM was organized to highlight and encourage recent advances in this topic. Each submitted paper was reviewed by experts in the area. Based on the critical reviews, we selected 7 papers to include in this special issue. They all contribute innovative ideas for the design and implementation of MIMO-OFDM based transceivers and networks that will meet the requirements of future generations of mobile communications. The first three papers cover the topics of channel quality estimation and performance prediction. With this information it is possible to perform adaptation at the transmitter, which is the topic of the fourth paper. The final two papers extend this possibility to multiuser and cooperative systems while the last paper proposes physical layer security techniques for MIMO-OFDM.

MIMO-OFDM systems use a cyclic prefix (CP) longer than the expected channel impulse response to maintain orthogonality at the expense of a small capacity loss. An insufficient CP may appear in some wireless scenarios with a very long channel response or in some military applications that suppress the $\mathrm{CP}$ to avoid signal identification.
The first paper "Joint Channel and Frequency Offset Estimation in MIMO-OFDM Systems with Insufficient Cyclic Prefix" [1] addresses joint channel and frequency offset estimation for MIMO-OFDM systems in the presence of Inter Symbol Interference and Inter Carrier Interference due to an insufficient $\mathrm{CP}$.

The second paper "CQI Algorithm Design in MIMO Systems with Maximum Likelihood Detectors" [2] investigates the problem of choosing a channel quality indicator (CQI) for individual streams in a $2 \times 2$ MIMO system, where the goal is to maximize the total throughput when data is scheduled based on the CQI chosen. Based on an information-theoretic analysis, a novel CQI algorithm is proposed and is shown to yield a throughput improvement in High Speed Downlink Packet Access (HSDPA), where MIMO-OFDM is used.

Performance prediction in practical MIMO-OFDM wireless links is challenging when forward error correction and interleaving are performed over subcarriers and spatial streams in frequency/spatial selective channels. Recent results show that the error-rate of coded and bit-interleaved MIMO-OFDM links may be characterized by the postprocessing signal-to-noise ratio (SNR), sorted over subcarriers, what is denoted as ordered SNR. The third paper "Modeling Ordered Subcarrier SNR in MIMO-OFDM Wireless Links" [3] establishes fundamental structure of this ordered SNR, obtaining interesting results that find several applications such as high-resolution limited channel feedback, simpler channel models for system simulation and algorithm design, and the reduction of dimension in link quality metrics for link adaptation.

Multicast transmission is an attractive way to provide common information like video simultaneously to multiple users. In conventional approaches to multicast, information is sent either at the most reliable mode of operation supported by the system, or is adapted to the performance of the worst user. The fourth paper "Distributed Link Adaptation for Multicast Traffic in MIMO-OFDM Systems" [4] shows how link adaptation can be used in multicast transmission to further increase data rates exploiting channel feedback from the users. It uses a data driven machine learning approach to propose a joint link adaptation 
algorithm that provides higher performance than individual adaptation.

The fifth paper "Minimum Bit Error Rate Nonlinear Precoding for Multiuser MIMO and High SNR" [5] focuses on the multiuser scenario, where MIMO allows several users to be served with the same space and time/frequency resources. Once full channel state information at the transmitter is available, a precoder is designed to separate the user signals at the transmitter side, canceling the multiuser interference, with the goal of minimizing the biterror-rate for the downlink of a multiuser MIMO system.

Cooperative relay networks have been proposed to exploit spatial diversity and improve network performance with the cooperation of multiple single-antenna terminals. With partial or full knowledge of channel state information, distributed beamforming can efficiently improve the capacity and reliability of relay networks. The sixth paper "Cooperative Beamforming for OFDM-based amplify-andforward Relay Networks" [6] investigates the cooperative beamforming design problem for amplify-and-forward relay networks that are operating over frequency-selective channels using OFDM signaling, with the aim of maximizing the minimum SNR over all subcarriers at the destination, using convex optimization tools.

Wireless networks are vulnerable to eavesdropping due to their broadcast nature. The way to guarantee secrecy is currently based on computationally demanding cryptographic algorithms implemented in the upper layers of the communication stack. As an alternative to these complex cryptographic techniques, physical layer security has recently emerged as a way to augment the system security by exploiting the spatio-temporal variations of the wireless channel. The seventh paper "Physical Layer Security of MIMO-OFDM Systems by Beamforming and Artificial Noise Generation" [7] addresses physical layer security in MIMO-OFDM frequency selective wireless channels where spatial beamforming and artificial noise broadcasting are leveraged to secure transmission.

In summary, this special issue highlights recent results in MIMO-OFDM that pave the way to new applications that can benefit from the multipath resilience and high throughput offered by these techniques. Lastly, we would like to thank Prof. Ian F. Akyildiz, Editor-in-Chief, and Devaprakash Kothandapani, Journal Manager, for helping us to organize the special issue. We also thank all authors and reviewers who contributed to the quality of the papers. We believe that this special issue is stimulating and presents a number of challenges and opportunities for future work.

\section{References}

[1] C. Prieto del Amo, V.P. Gil Jiménez, M.J. Fernández-Getino García, Joint channel and frequency offset estimation in MIMO-OFDM systems with insufficient cyclic prefix, PHYCOM SI-Advances in MIMO-OFDM 4 (4) (2011) 254-265.

[2] Y. Su, J. Tang, G. Mergen, P. Subrahmanya, L. Xiao, J. Sidi, A. Salvekar, CQI algorithm design in MIMO systems with maximum likelihood detectors, PHYCOM SI-Advances in MIMO-OFDM 4 (4) (2011) 266-274.

[3] R.C. Daniels, R.W. Heath Jr., Modeling ordered subcarrier SNR in MIMO-OFDM wireless links, PHYCOM SI-Advances in MIMO-OFDM 4 (4) (2011) 275-285.
[4] S. Yun, C. Caramanis, R.W. Heath Jr., Distributed link adaptation for multicast traffic in MIMO-OFDM systems, PHYCOM SI-Advances in MIMO-OFDM 4 (4) (2011) 286-295.

[5] D. Castanheira, A. Gameiro, A. Silva, Minimum bit error rate nonlinear precoding for multiuser MIMO and high SNR, PHYCOM SI-Advances in MIMO-OFDM 4 (4) (2011) 296-304.

[6] W. Cheng, M. Ghogho, Q. Huang, D. Ma, J. Wei, Cooperative beamforming for OFDM-based amplify-and-forward relay networks, PHYCOM SI-Advances in MIMO-OFDM 4 (4) (2011) 305-312.

[7] N. Romero-Zurita, M. Ghogho, D. McLernon, Physical layer security of MIMO-OFDM systems by beamforming and artificial noise generation, PHYCOM SI-Advances in MIMO-OFDM 4 (4) (2011) 313-321.

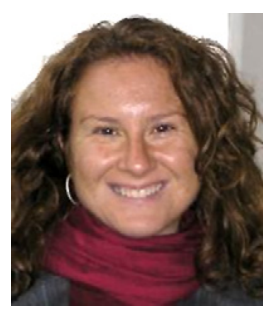

Ana García Armada received the Ph.D. degree in electrical engineering from the Polytechnic University of Madrid in February 1998. She is currently an Associate Professor with the University Carlos III of Madrid, where she has occupied a variety of management positions and is now acting as Deputy Vice-chancellor of International Relations. She is leading the Communications Research Group of this university and the Laboratory of Communication Systems for Security and Space in the Scientific Park of the same university. She has participated in several national and international research projects related to wireless communications. She is the coauthor of 8 book chapters on wireless communications and signal processing. She has published more than 30 papers in international journals and more than 50 contributions to international conference proceedings. She has contributed to international organizations such as ITU and ETSI. Her main interests are OFDM and MIMO techniques and their application to wireless communications. She has received several awards from University Carlos III of Madrid including the Excellence Award to a young researcher's trajectory and the award to Best Practice in lecturing activities.

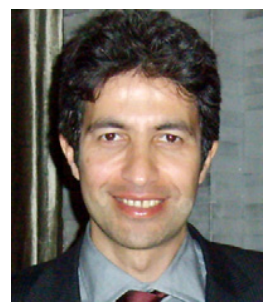

Mounir Ghogho received the M.S. degree in 1993 and the Ph.D. degree in 1997 from the National Polytechnic Institute of Toulouse, France. He was an EPSRC Research Fellow with the University of Strathclyde, Glasgow, from September 1997 to November 2001. Since December 2001, he has been a faculty member with the school of Electronic and Electrical Engineering at the University of Leeds, where he is currently a Professor. He is also currently a Professor at the International University of Rabat in Morocco. He served as an Associate Editor of the IEEE Signal Processing Letters from 2001 to 2004 and the IEEE Transactions on Signal Processing from 2005 to 2008. He also served as a member of the IEEE Signal Processing Society SPCOM Technical Committee from 2005 to 2010 and is currently a member of the IEEE Signal Processing Society SPTM Technical Committee. He was the general co-chair of the eleventh IEEE workshop on Signal Processing for Advanced Wireless Communications (SPAWC'2010), the technical co-chair of the MIMO symposium of IWCMC 2007 and IWCMC 2008, and a technical area cochair of Eusipco 2008, Eusipco 2009 and ISCCSP'05. He is the general chair of Eusipco 2013. He was the guest co-editor of the EURASIP Journal on Wireless Communications and Networking special issue on "synchronization for wireless communications". His research interests are in communication and sensor networks, radar imaging and signal processing. He was awarded a five-year Royal Academy of Engineering Research Fellowship in September 2000.

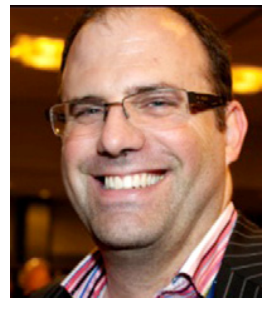

Robert W. Heath Jr. received the Ph.D. degree in electrical engineering from Stanford University in 2002. He is currently an Associate Professor at The University of Texas at Austin. He is also President and CEO of MIMO Wireless Inc. and VP of Innovation at Kuma Signals LLC. He has been an Editor for the IEEE Transactions on Communication, an Associate Editor for the IEEE Transactions on Vehicular Technology, and lead guest editor for several special issues in journals. He was a member of the IEEE Signal Processing 
Society SPCOM Technical Committee. He is Chair of the IEEE COMSOC Communications Technical Theory Committee. He was a co-author of several best student paper awards at conferences and was co-recipient of the 2010 EURASIP Journal on Wireless Communications and Networking best paper award. He is the recipient of the David and Doris Lybarger Endowed Faculty Fellowship in Engineering. He is a licensed Amateur Radio Operator, a registered Professional Engineer in Texas, and is a Fellow of the IEEE.

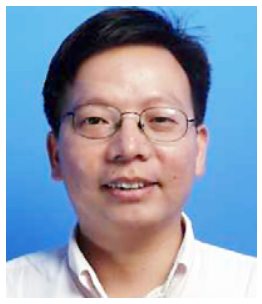

Ying-Chang Liang is a Principal Scientist with the Institute for Infocomm Research (I2R), Agency for Science, Technology \& Research (A*STAR), Singapore. He was a visiting scholar in the Department of Electrical Engineering, Stanford University, CA, USA, from Dec 2002 to Dec 2003, and taught graduate courses in National University of Singapore from 2004-2009. He was elected a Fellow of the IEEE in 2011, and received Best Paper Awards from IEEE VTC-Fall in 1999, IEEE PIMRC in 2005, IEEE VTC-Spring in 2011, and EURASIP Journal of Wireless Communications and Networking in 2010. He also received the Institute of Engineers Singapore (IES)'s Prestigious Engineering Achievement Award in 2007, I2R's Achiever of the Year Award in 2008, and the IEEE Standards Association's Outstanding Contribution Appreciation Award in 2011, for his contributions to the development of IEEE 802.22, the first worldwide standard based on cognitive radio technology.

Liang currently serves as Editor-in-Chief of IEEE Journal on Selected Areas in Communications - Cognitive Radio Series, and Associate Editor of IEEE Transactions on Vehicular Technology. He was an Associate Editor of IEEE Transactions on Wireless Communications from 2002 to 2005, and served as a Guest Editor of five special issues on emerging topics published in IEEE, EURASIP and Elsevier journals in the past five years. He is currently a Distinguished Lecturer of the IEEE Vehicular Technology Society, and has been a member of the Board of Governors of the IEEE Asia-Pacific Wireless Communications Symposium since 2009. He is the Wireless Communication Symposium Chair of 2012 IEEE International Conference on Communications (ICC'12), and Chair of 2012 Thematic Program on random matrix theory and its applications, organized by Institute for Mathematical Sciences, National University of Singapore.

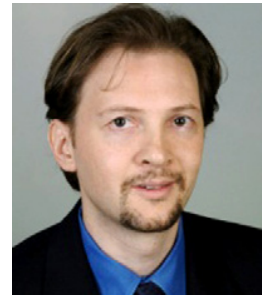

Constantinos Papadias (IEEE SM'03) is a Professor at Athens Information Technology (AIT), in Athens, Greece, as well the Academic Director of AIT's Doctoral Program, which runs in cooperation with Aalborg University in Denmark. He received the Diploma of Electrical Engineering from the National Technical University of Athens (NTUA) in 1991 and the Doctorate degree in Signal Processing (highest honors) from the Ecole Nationale Supérieure des Télécommunications (ENST), Paris, France, in 1995. He was a researcher at Institut Eurécom, Stanford University and Bell Labs and an Adjunct Professor at Columbia University and Carnegie Mellon University. His research interests span several areas of advanced communications systems, with emphasis on wireless, cognitive, green and next generation networks. He has published over 130 papers, 5 book chapters, one edited book, and has received over 3000 citations for his work. His distinctions include the 2002 Bell Labs President's Award, a Bell Labs Teamwork Award, the 2003 IEEE Signal Processing Society's Young Author Best Paper Award and ESI's "most cited paper of the decade" citation in the area of wireless networks in 2006. He has also made standards contributions (most notably as the co-inventor of the Space-Time Spreading (STS) technique that was adopted by the cdma2000 wireless standard for voice transmission) and holds 12 patents. He is currently the Technical Coordinator of two EU FP7 FET research projects: CROWN, in the area of cognitive radio systems and HIATUS, in the area of interference alignment. He is a co-author of the book "MIMO Communication for Cellular Networks" which was recently published by Springer.

Atul Salvekar received a Ph.D. degree in Electrical Engineering from Stanford University in 2002. He is currently a senior staff/manager at Qualcomm. He works on all aspects of physical layer modem design across $2 \mathrm{G} / 3 \mathrm{G} / 4 \mathrm{G}$ cellular standards. He is the author of 4 book chapters, 4 journal papers, 9 conference papers, and 4 patents with several more pending.

\section{Ana García Armada Mounir Ghogho Robert W. Heath Jr. Ying-Chang Liang Constantinos Papadias Atul Salvekar}

